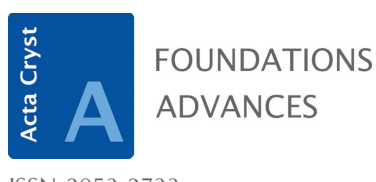

ISSN 2053-2733

\title{
Quantum Field Theory Approach to Condensed Matter Physics. By Eduardo C. Marino. Cambridge University Press, 2017. Hardback, pp. 532. Price GBP 64.99. ISBN 9781107074118.
}

\author{
Malte Henkel*
}

Université de Lorraine, CNRS, LPCT, Nancy, France, and Centro de Física Téorica e Computacional, Universidade de Lisboa, Lisboa, Portugal. *Correspondence e-mail: malte.henkel@univ-lorraine.fr

Keywords: book review; quantum field theory; condensed matter physics.

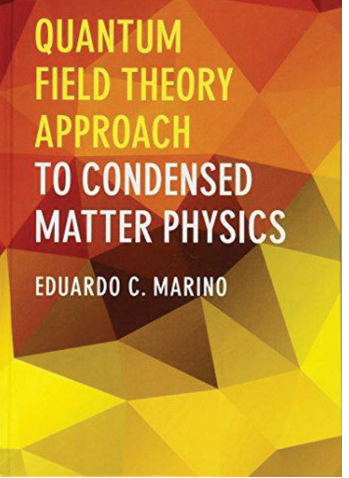

(C) 2019 International Union of Crystallography
Quantum field theory has become an indispensable tool in many areas of physics. In his book Quantum Field Theory Approach to Condensed Matter Physics, Eduardo Marino strives not so much at the development of fundamental issues in the abstract theory of quantum field but rather at how to make known concepts and techniques useful for theoretical condensed matter physics. The scope is limited to systems at thermal equilibrium, or at most to linear response around the equilibrium state. Certainly, this is not the first attempt of its kind. However, timely summaries of new methods and how they arise from older concepts can provide a useful entry point to newcomers in the field, be they graduate students, young post-docs or even specialists from other fields who wish an overview of present-day field theory methods applied in condensed matter theory. The author is to be lauded for having undertaken the vast enterprise of bringing together, in a not too unwieldy volume, much of the basics, well illustrated by several applications chosen from a truly enormous range. I shall certainly use the book to look up details I should have known but which may have slipped from my memory.

In order to make this work sufficiently self-contained and therefore accessible to the graduate student, the first part of the book is devoted to a rapid reminder of some basic elements of properties of the solid state. The Bloch theorem, vibrating crystals, interacting electrons along with Hubbard and Heisenberg models and the ferromagnetic and superconducting phase transitions are briefly reviewed. The presentation may be a bit too dense for a complete newcomer but for all those who took a class in theoretical solid-state physics, a reminder of this kind will be welcome. In a similar spirit, the second part treats the basics of quantum field theory and refreshingly starts with an introduction to functionals and functional integrals. A little provocatively, the 'whole physics' is presented in only three formulae. From this functional foundation, perturbation theory, functional determinants, renormalization and the renormalization group are rapidly introduced, up to the Callan-Symanzik equation. Special sections cover both classical and quantum topological excitations. A speciality of the book is the detailed treatment of pseudo quantum electrodynamics, with the full three-dimensional electromagnetic field but where the motion of the particles is confined to the plane, and with its distinctive spatially non-local Lagrangian. As in the first part, the presentation is rapid and compact. For a knowledgeable reader, this will be a welcome reminder, but for the novice in particular the second part will present a challenge. In particular, although the main abstract results are well composed and clearly exposed, the presentation lacks explicitly worked-out examples, very much in contrast with the classic textbook of Altland \& Simons, Condensed-Matter Field Theory, of a comparable scope. For example, not a single case study illustrates the formulation and use of the Callan-Symanzik equation and its associated $\beta$ - and $\gamma$-functions, and how stable and unstable fixed points are obtained from the zeroes of the $\beta$-function. Also, in the treatment of finite-temperature field theory, why is there no mention at all of the possible geometric interpretation of $\hbar \beta$ as an inverse length scale of a system in an extra spatial dimension? Here, inspiration from a text oriented towards high-energy physics, such as Wipf's Statistical Approach to Quantumfield Theory and the avenues which that work describes, would have been helpful. And finally, why not re-derive Schrödinger's equation from the functional integral used to 
define quantum field theory? Students in particular would welcome seeing how the 'new' formalism can be matched with an 'old' one.

The last and main part covers more than half of the book and reviews advanced applications of the techniques presented before to physical problems in condensed matter physics. The incredible breadth of the topics treated (for a volume of a little more than 500 pages) includes Mott and Anderson isolators, polarons, polyacetylene, the Kondo effect, one- and two-dimensional quantum magnets, spin glass superfluidity, superconductivity of cuprates and pnictides, graphene and transition metal dichalcogenides, the quantum Hall effect, and of course topological insulators and quantum computation. Each of the chapters begins with a short introduction and motivation before the topic itself is carefully explained, usually starting quite elementarily. Certainly, the chapters of Part 3 give an accessible entry into the literature for a long and broad list of active research topics in theoretical condensed matter physics. The other feature of the book is that it is exclusively oriented towards analytical treatments and numerical methods are not covered. Experimental results are occasionally mentioned in passing.

An experienced teacher could use this as a textbook for a class on field theory methods applied to condensed matter physics, but I suspect one would feel the need to look elsewhere for a sufficient number of explicitly worked-through examples for illustration of the general principles. The enormous breadth of the topics treated, notably in Part 3, will certainly permit one to select a few of them for more detailed presentation. Similarly, the volume could also be used by a non-specialist as a reference in order to get a quick overview on current topics, but I suspect that for details the original literature would have to be consulted. With five pages, the index is relatively detailed but still too short for a reference volume. Reading the text is smooth and pleasant, but as with many works in the field, the book suffers from the unfortunate habit of denoting a function and its Fourier transform by the same letter and distinguishing them merely by the name of their respective arguments. Besides being mathematically meaningless, such a convention is unnecessarily confusing.

In summary, this is a useful book to have in one's library. 